\title{
Diseño e implementación de un controlador proporcional integral en un controlador industrial de procesos
}

\begin{abstract}
RESUMEN
El presente trabajo presenta una metodología de diseño de un controlador con estrategia proporcional integral (PI) teniendo en cuenta que la implementación se realizará en un controlador industrial de procesos. El diseño considera la modelación de la planta mediante técnicas analógicas de identificación de sistemas. Se utiliza en el diseño del controlador el algoritmo digital del controlador discreto PI que ejecuta el controlador de procesos utilizado. Se consideran para el diseño especificaciones de respuesta temporal de lazo cerrado básicamente para obtener un tiempo de estabilización acorde a los que puede cumplirse sin exceder los rangos de la señal de control que puede entregar el controlador de procesos utilizado. Se implementa el sistema de control verificando el comportamiento del sistema de lazo cerrado en relación al objetivo de control especificado.

Palabras clave: Sistemas de lazo cerrado, controlador de procesos, identificación de sistemas, algoritmo de control, modelos de sistemas, diseño de controladores.
\end{abstract}

DESIGN AND IMPLEMENTATION OF A Proportional Integral Controller in Controller Industrial Process

\section{ABSTRACT}

This paper presents a methodology for designing a strategy proportional integral controller (PI) given that the policy will be implemented in an industrial process controller. The design considers the modeling of the plant through analog techniques of system identification. It is used in controller design algorithm discrete digital PI controller that is running the process controller used. Considered for the design specifications of closed loop time response primarily to obtain a settling time line that you can be met without exceeding the range of the control signal that can deliver the process controller used. Implement the control system to verify the behavior of the closed loop system in relation to the specified control objective.

Keywords: Closed loop systems, process control system identification, control algorithm, system models, design of controllers.

\section{INTRODUCCIÓN}

En los procesos industriales de fabricación es de vital importancia el control de variables continuas (temperatura, presión, nivel, flujo, etc.) en las diferentes partes de la línea de producción, siendo clave en el desempeño de cada subproceso el mantener en condiciones estándar las variables continuas involucradas en él. La calidad del producto final refleja qué tan bien el proceso controla las variables continuas en las diferentes etapas que lo constituyen. Cada lazo de control de las variables continuas consideradas debe diseñarse con cierta estrategia que permita, al trabajar en conjunto todos los sistemas de lazo cerrado, asegurar el desempeño del sistema total. En los procesos de producción es común utilizar un controlador industrial de procesos como instrumento que se encarga de controlar las variables analógicas involucradas en los procesos. También la mayoría de ellos ofrece una configuración del clásico controlador de componentes proporcional $(P)$, integral $(I)$ y derivativa (D) $[1,2]$, mediante una determinada ley de control digital que no necesariamente el diseñador la conoce de antemano o el fabricante la ofrece. Así, el personal responsable de planta para controlar las variables del proceso debe recurrir a la experiencia de los que conocen el proceso o al empirismo, con la finalidad de manejar sus procesos adecuadamente. Se desarrolla una metodología que servirá para diseñar un controlador de componentes proporcional integral a ser implementado en el controlador industrial Hanyoung NX9 [3], para controlar un proceso industrial. Se toma en cuenta las características de este controlador en cuanto a los modos y rangos de entrada aceptable, tipos y rangos de salida que ofrece, escalas de entrada configurables, rangos de los parámetros de sintonía de la estrategia PID, tiempo de muestreo de las entrada analógicas, la señal de entrada manual que se puede aplicar en lazo abierto, etc. Se estudia los modos proporcional e integral en forma independiente para deducir la salida de control en cada caso y su relación con los parámetros de banda proporcional y tiempo integral que son los valores a los cuales el operador del instrumento tiene acceso para modificar el comportamiento del controlador. Esos resultados sirven para definir la estructura de la ley de control digital que se utiliza como estructura del controlador digital diseñado.

Magíster en Ingeniería de Control. Docente de la Facultad de Ingeniería Electrónica y Eléctrica, Universidad Nacional Mayor de San Marcos, Lima, Perú.

E-mail: bvargast@unmsm.edu.pe 


\section{ANÁLISIS Y OBTENCIÓN DEL DIAGRAMA DE BLOQUES DE LAZO CERRADO A IMPLEMEN- TARSE UTILIZANDO EL CONTROLADOR DE PROCESOS HANYOUNG NX9}

A. Definición de parámetros configurables en el controlador de procesos Hanyoung NX9

El controlador de procesos Hanyoung NX9 dentro de sus diferentes menús configurables permite asignar valores y opciones a diferentes parámetros, indicamos aquellos que son necesarios para la implementación de un controlador proporcional integral.

SLH: valor máximo de la escala del rango de entrada de la variable del proceso que se controlará

SLL: valor mínimo de la escala del rango de entrada de la variable del proceso que se controlará

SV: valor de setpoint o de referencia deseado para la variable del proceso (dentro del rango de escala de entrada configurado), este valor es mostrado en el display del controlador.

PV: valor actual que recibe el controlador de la variable del proceso (en el rango de entrada configurado), este valor es mostrado en el display del controlador.

IPH: valor máximo de la señal de entrada conectada al controlador.

IPL: valor mínimo de la señal de entrada conectada al controlador

PB: banda proporcional (proporción de la señal de control que entrega el controlador cuando recibe una entrada que produce un error de $100 \%$ en relación con el rango de entrada que configurado, cuando sólo se utiliza componente proporcional).

TI: tiempo integral (tiempo que requiere el controlador para producir el mismo valor de señal de control que genera la componente proporcional, para el mismo valor del error).

TS: tiempo de muestreo especificado por el fabricante para la adquisición de datos analógicos

MR: porcentaje de la señal de control que entrega el controlador antes de pasar a modo automático.

$\triangle O U T:$ cambio respecto al valor MR de la señal de control que calcula el controlador de acuerdo a la estrategia configurada

OUT: valor de la señal de control que calcula el controlador en relación al rango de salida (este valor es mostrado en el display del controlador).
El controlador debe recibir en una de sus entradas la señal de los sensores de la variable controlada que se encuentra en el rango especificado por SLL y SLH. El sensor debe tener una salida en el rango aceptable por el controlador. En este caso, en la opción del menú de configuración G.In configuramos al valor de 30 , el cual indica que la entrada que recibirá el controlador está en el rango de 1 a 5 voltios. En esa opción, se configura IPH a 5V y IPL a 1V.

El controlador entregará una señal de control de rango industrial estándar que recibirá la planta. En nuestro caso, en la opción del menú de configuración G.Out se configura al valor de 2, el cual indica que salida de control entregará una señal de corriente en el rango de 4 a $20 \mathrm{~mA}$.

B. Cálculo de los valores que utiliza el controlador para ejecutar el algoritmo de control y obtención de la salida de control a partir de la señal de control calculada por el algoritmo de control

Sea y(t) la señal del sensor que se conecta a la entrada del controlador industrial. El controlador lee un valor de entrada $y(t)$ que debe estar en el rango de entrada de IPL a IPH. El valor de y(t) debe convertirse a un valor en el rango de la escala de la variable del proceso a controlarse de SLL a SLH y es el llamado valor del proceso PV. La figura 1, muestra el proceso de obtención de la variable de proceso PV que realiza el controlador

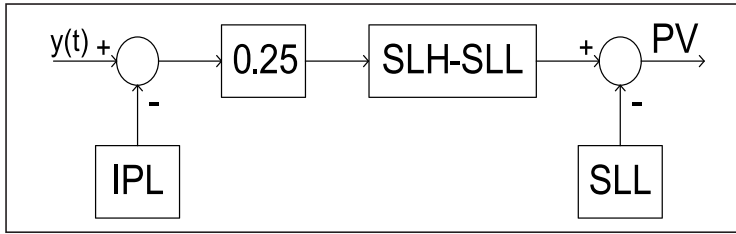

Fuente : Elaboración propia.

Figura 1. Obtención del valor PV.

Configurados los parámetros del controlador, este calcula previamente el valor $\triangle O U T$ de acuerdo a la estrategia de control elegida, al valor de SV y al valor calculado de PV. Luego debe calcular el valor OUT y convertirlo a una corriente de salida de control que produce el controlador en sus terminales de salida y que se encuentra en el rango de 4 a $20 \mathrm{~mA}$. Sea $\mathrm{I}_{\mathrm{o}}$ el valor de la corriente de salida que entrega el controlador, la figura 2, muestra como se obtiene el valor de I a la salida del controlador. 


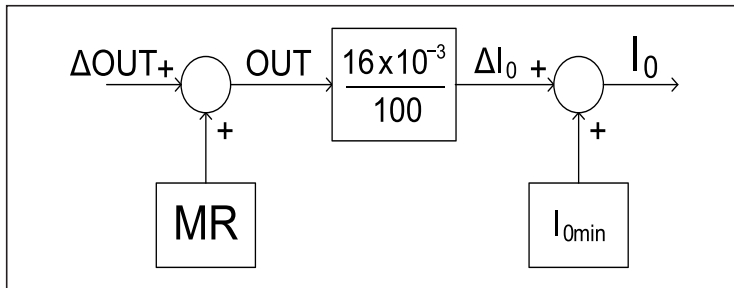

Fuente: Elaboración propia.

Figura 2. Obtención de la corriente de salida del controlador.

C. Modelo requerido para la planta a ser utilizado en el diseño del controlador

Según se observa en las figuras 1 y 2 , el controlador recibirá como entrada la señal $\mathrm{y}(\mathrm{t})$ y entregará la señal de salida $I_{0}(t)$, por tanto el modelo de la planta a utilizarse en el diseño del controlador debe considerar a $\mathrm{I}_{0}(\mathrm{t})$ como entrada y a $y(t)$ como salida. Considerando que es común establecer un valor de consigna deseado para la salida de la planta, llamado punto de operación podemos utilizar el valor MR configurable para que el controlador en lazo abierto lleve a la salida a ese valor de consigna y que en lazo cerrado, cuando se produzcan perturbaciones el controlador genere la señal $\triangle$ OUT que produzca un valor $\Delta \mathrm{l}_{\mathrm{o}}(\mathrm{t})$ para compensar el efecto perturbador y permita mantener la salida en el valor deseado. Como la dinámica del controlador hará variar la salida respecto al punto de operación, el modelo a utilizar de la planta para el diseño del controlador, debe ser aquél que represente las variaciones de salida para variaciones de entrada de la planta respecto al punto de operación. Sea $y_{O P}$ el valor de salida de operación para $u_{\mathrm{OP}}$ el valor de entrada de operación, entonces si $\Delta y(t)$ representa la variación del valor de salida $\mathrm{y}(\mathrm{t})$ respecto $a \mathrm{y}_{\mathrm{OP}} \mathrm{y} \Delta \mathrm{u}(\mathrm{t})$ representa la variación del valor de entrada $\mathrm{u}(\mathrm{t})$ respecto a $\mathrm{u}_{\mathrm{op}}$, entonces la Figura 3, muestra como se obtiene la señal de salida $y(t)$ en función de $u(t)$.

Ya que las variaciones de $\Delta y(t)$ son debidas a las variaciones $\Delta \mathrm{u}(\mathrm{t})$, se puede realizar un procedimiento con pruebas experimentales para obtener un modelo de función de transferencia $\mathrm{G}(\mathrm{s})$ que represente las variaciones de salida por efecto de las variaciones de entrada de la planta. Ese es el llamado proceso de identificación de sistema analógico. La Figura 4, representa en un bloque el modelo de la planta respecto al punto de operación.

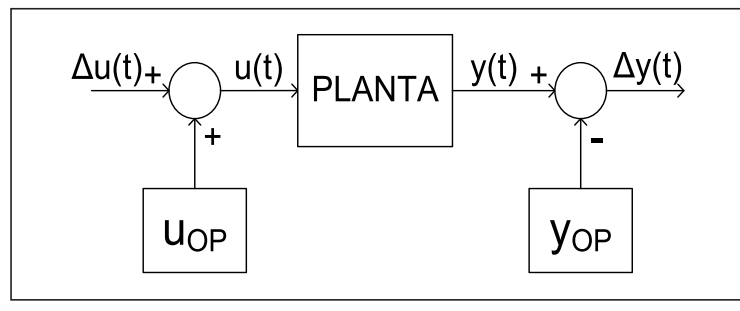

Fuente: Elaboración propia.

Figura 3. Representación de la planta alrededor del punto de operación.

\section{$\frac{\Delta \mathrm{u}(\mathrm{t})}{\Delta \mathrm{u}(\mathrm{s})} \mathrm{G}(\mathrm{s}) \frac{\Delta \mathrm{y}(\mathrm{t})}{\Delta \mathrm{y}(\mathrm{s})}$}

Fuente: Elaboración propia.

Figura 4. Modelo de la planta alrededor del punto de operación.

D. Diagrama de bloques de lazo cerrado utilizado para el diseño del controlador digital

Obtenido el modelo de la planta luego de la identificación, la función de transferencia G(s) que representa la planta se utiliza para el diseño del controlador digital, considerando la estructura de lazo cerrado con control digital en cascada con la planta antepuesta por un circuito de retención de orden cero $(\mathrm{ZOH})$, el diagrama de bloques de lazo cerrado considerado para el diseño del controlador digital se muestra en la Figura 5.

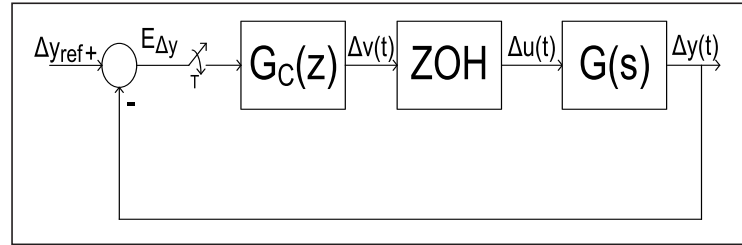

Fuente: Elaboración propia.

Figura 5. Diagrama de bloques de lazo cerrado utilizado para el diseño del controlador digital.

En este caso se considera $\Delta y_{\text {ref }}$ como el valor de referencia deseado para $\Delta y$. El controlador digital diseñado se representa por su función de transferencia $G_{c}(z)$ discreta. 
E. Diagrama de bloques del sistema de lazo cerrado a implementar

Considerando a la planta real cuya señal de salida $\mathrm{y}(\mathrm{t})$ será realimentada para implementar el sistema de lazo cerrado, tendremos a partir de la Figura 3 que el diagrama de bloques a implementar en lazo cerrado que es equivalente a la Figura 5, sería el representado en la Figura 6.

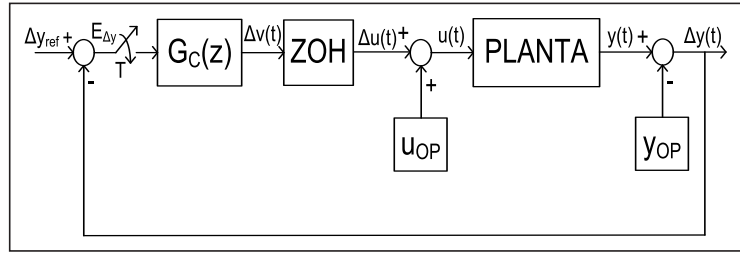

Fuente: Elaboración propia

Figura 6. Diagrama de bloques de lazo cerrado considerando a la planta real a ser controlada.

En razón que $\Delta \mathrm{y}_{\text {ref }}$ es el cambio del valor de referencia deseado para $\Delta y$ y teniendo en cuenta que $\Delta y$ considera la variación de salida respecto al punto de operación $\mathrm{y}_{\mathrm{OP}}$, entonces el valor $\Delta \mathrm{y}_{\text {ref }}$ también está referenciado respecto a $\mathrm{y}_{\mathrm{OP}}$. Si la entrada de referencia $y_{\text {ref }}$ representa el valor deseado en la salida $y(t)$, entonces se cumple que:

$$
\Delta y_{\text {ref }}=y_{\text {ref }}-y_{O P}
$$

Utilizando (1) el diagrama de bloques de la figura 6 , tiene el equivalente en diagrama de bloques que se muestra en la figura 7 .

\section{DIAGRAMA DE BLOQUES DEL SISTEMA DE LAZO CERRADO CONSIDERANDO LOS PARA- METROS DE SINTONÍA DEL CONTROLADOR DE PROCESOS HANYOUNG NX9 EN CONFIGU- RACIÓN PROPORCIONAL INTEGRAL}

El diagrama de bloques de la Figura 7 implementará el controlador $\mathrm{G}_{c}(\mathrm{z})$ utilizando la sintonía de los parámetros de banda proporcional PB y tiempo integral TI del controlador de procesos Hanyoung NX9. En función de los parámetros mencionados el controlador utiliza la señal de error que calcula en relación al rango de señal del proceso configurada (ese rango configurado varía de SLL a SLH).

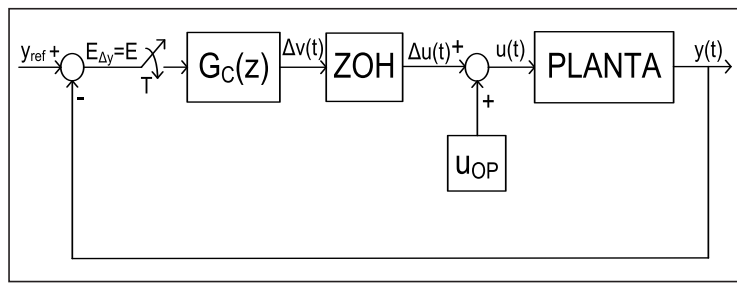

Fuente : Elaboración propia.

Figura 7. Diagrama de bloques de lazo cerrado considerando la entrada de referencia deseada de la salida.

El diagrama de bloques que describe la obtención de la señal de control que se agrega al valor de entrada manual MR se muestra en la Figura 8.

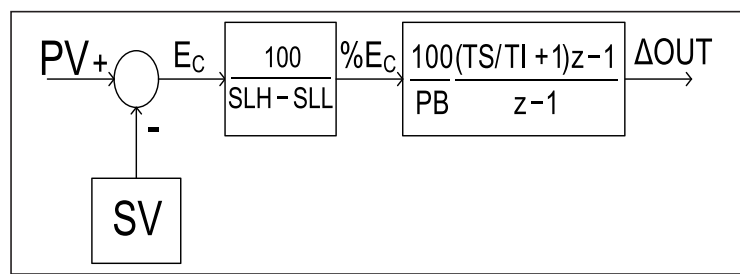

Fuente: Elaboración propia

Figura 8. Obtención de la salida que calcula el algoritmo de control PI del controlador de procesos utilizado.

\section{DIAGRAMA DE BLOQUES DEL SISTEMA DE LAZO CERRADO IMPLEMENTADO CON EL CONTROLADOR DE PROCESOS Y EL DIAGRA- MA DE BLOQUES PARA EL DISEÑO DEL CON- TROLADOR}

Combinando las Figuras 8, 3 y 1, se obtiene el diagrama de bloques del instrumento controlador de procesos desde su entrada (salida del proceso a controlar) hasta la obtención de la señal de control que se aplica a la entrada del proceso. El diagrama de bloques mencionado se muestra en la Figura 9.

La señal de corriente configurada como salida circulará por una resistencia $\mathrm{R}$ de 250 ohmios para convertirla a una tensión en el rango de 1 a 5 voltios.

El diagrama de bloques equivalente que se obtuvo a partir de la Figura 9, se muestra en la Figura 10. 


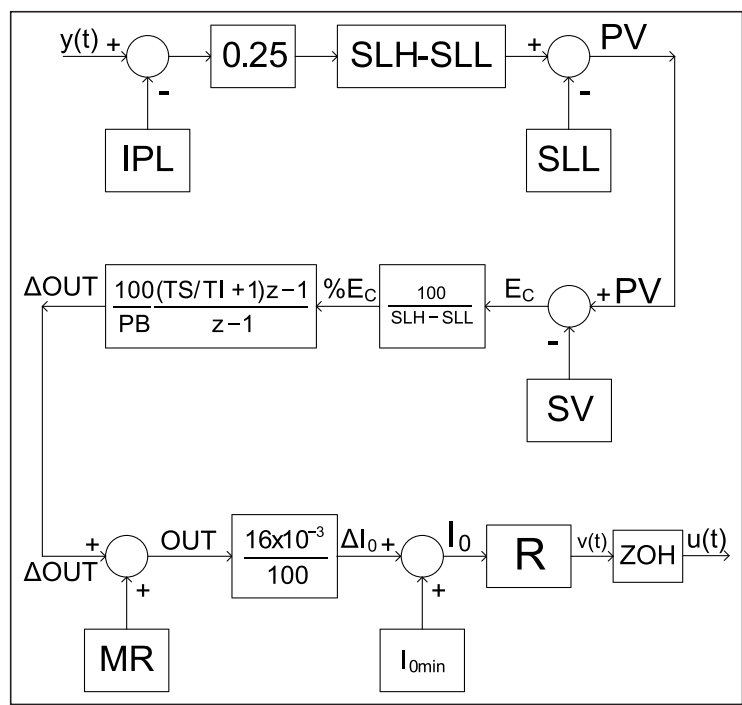

Fuente : Elaboración propia.

Figura 9. Diagrama de bloques del instrumento controlador.

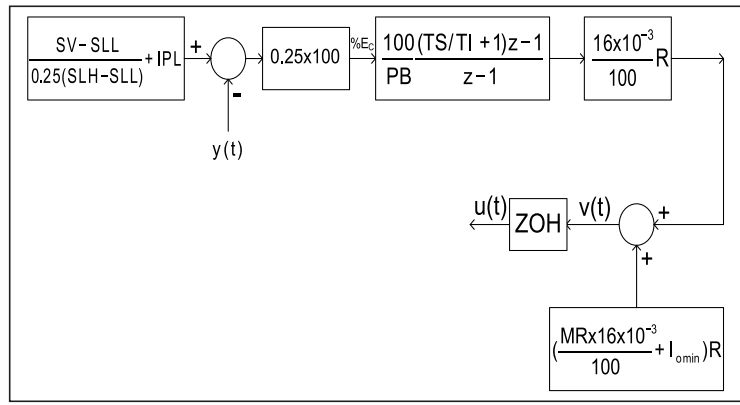

Fuente : Elaboración propia.

Figura 10. Diagrama de bloques equivalente del instrumento controlador al de la figura 9.

Considerando a la planta como el proceso que completa el sistema de lazo cerrado, entonces se agrega la planta en la Figura 10 y se obtiene el sistema de lazo cerrado que se muestra en la Figura 11.

Comparando las Figuras 11 y 7 se obtienen las siguientes relaciones:

$y_{r e f}=\frac{S V-S L L}{0.25(S L H-S L L)}+I P L$

$$
\begin{aligned}
& G_{C}(z)=0.25 \times 100 \frac{100}{P B} \frac{(T S / T I+1) z-1}{z-1} \times \frac{16 \times 10^{-3}}{100} R \\
& u_{O P}=\left(\frac{M R x 16 \times 10^{-3}}{100}+I_{o \text { min }}\right) R
\end{aligned}
$$

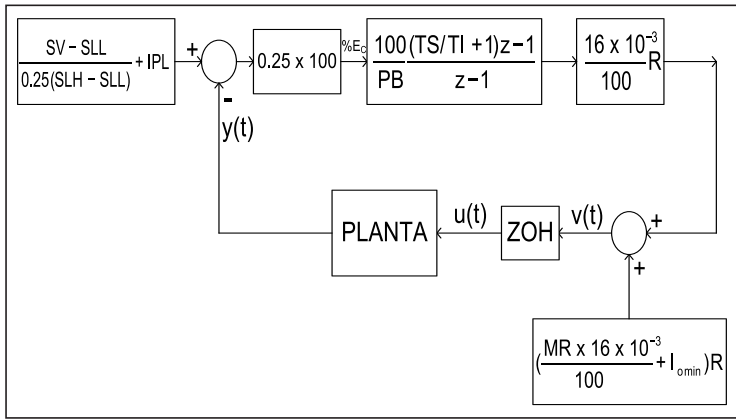

Fuente : Elaboración propia.

Figura 11. Diagrama de bloques equivalente de lazo cerrado que incluye el efecto de todos los parámetros configurables del controlador industrial utilizado.

\section{DISEÑO DEL CONTROLADOR DIGITAL PRO- PORCIONAL INTEGRAL (PI)}

Se utiliza el diagrama de bloques representado en la Figura 5, como base para el diseño del controlador digital proporcional integral (PI). Se inicia el proceso de diseño mediante la identificación analógica de la planta.

\section{A. Identificación analógica de la planta}

Para obtener el modelo de función de transferencia $\mathrm{G}(\mathrm{s})$ que represente las variaciones de salida por efecto de las variaciones de entrada de la planta (Figura 4), se aplica en lazo abierto una señal constante de entrada que estabilice la salida de la planta en una tensión que equivale al punto de operación de salida de 3 voltios. Luego se producen cambios de entrada para que la salida varíe alrededor de ese punto de operación. Se han registrado mediante un sistema de adquisición de datos los datos de entrada y salida de la planta que se muestran en las Figuras $12 \mathrm{a}$ y $12 \mathrm{~b}$. respectivamente. 


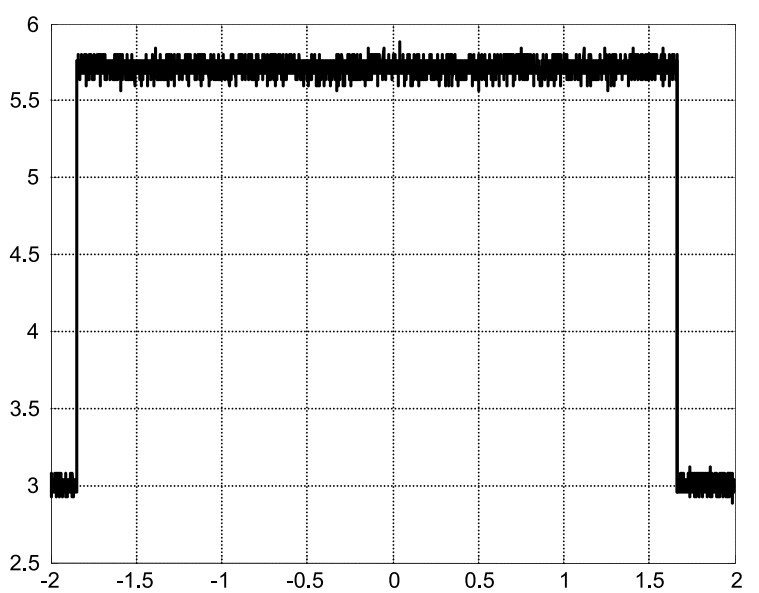

Fuente: Elaboración propia.

Figura 12a. Señal del tipo cambio escalón en la entrada de la planta de prueba respecto al punto de operación.

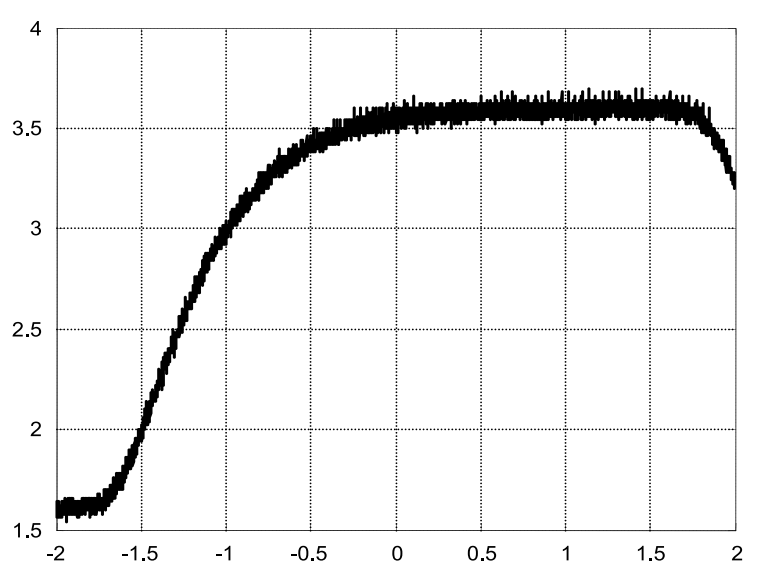

Fuente: Elaboración propia.

Figura 12b. Respuesta respecto al punto de operación de la salida para un cambio escalón en la entrada de la planta de prueba.

La Figura 12b. es la llamada curva de reacción del proceso a controlar. En base a los resultados de esa prueba, desarrollamos la identificación analógica de la planta utilizando el método de los tres puntos $[4,5]$ y obtenemos un modelo representativo de segundo orden sobreamortigua- do con retardo. El modelo de la planta obtenido es el siguiente:

$$
\begin{aligned}
& G(s)=\frac{7.69}{\mathrm{~s}^{2}+6.645 \mathrm{~s}+10.57} \mathrm{e}^{-0.102 \mathrm{~s}} \\
& G(s)=\frac{7.69}{(\mathrm{~s}+4.007)(\mathrm{s}+2.638)} \mathrm{e}^{-0.102 \mathrm{~s}}
\end{aligned}
$$

Para validar el modelo se realizan pruebas a la planta respecto al punto de operación. La respuesta del modelo para esas entradas registradas se comparan con las medidas, obteniéndose una gran aproximación en ambas respuestas. La Figura 13 muestra en un caso la respuesta medida y la respuesta del modelo, se observa que ambas están prácticamente superpuestas.

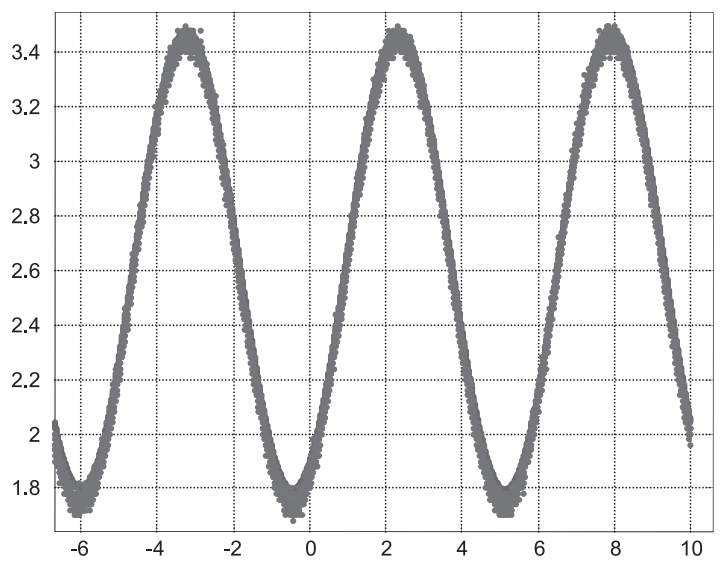

Fuente: Elaboración propia.

Figura 13. Respuesta del modelo y del proceso para la misma entrada de prueba (validación del modelo identificado).

\section{B. Modelo discreto de la planta con anteposi- ción de ZOH}

Para el diseño del controlador PI discreto, según la Figura 5, se utiliza el equivalente discreto de la planta con anteposición de $\mathrm{ZOH}$. En este caso el período de muestreo que indica el fabricante del controlador para la implementación de su algoritmo de control es de TS $=250 \mathrm{msg}$. El equivalente discreto que corresponde en este caso a la planta es: 


$$
\begin{aligned}
& G(z)=\frac{0.06115 z^{2}+0.1516 z+0.009556}{z\left(z^{2}-0.8844 z+0.1899\right)} \\
& G(s)=\frac{0.06115(z+2.414)(z+0.06473)}{z(z-0.5172)(z-0.3672)}
\end{aligned}
$$

\section{Estructura de función de transferencia del controlador proporcional integral discreto}

De acuerdo a la ecuación (3) dada una banda proporcional PB y un tiempo integral TI (los valores de TS y R están definidos), el controlador proporcional integral discreto $(\mathrm{PI})$ que se diseñará tiene la siguiente estructura de función de transferencia discreto:

$$
G_{P I}(\mathrm{z})=\mathrm{K} \frac{(\mathrm{z}-\mathrm{a})}{(\mathrm{z}-1)}
$$

El diseño de ese controlador se realizará considerando el diagrama de bloques de la figura 5 , luego se transformará a la forma de la ecuación 3, para obtener los valores de sintonía de PB y TI.

\section{Especificaciones del sistema de lazo cerrado}

El sistema de lazo cerrado (figura 7) debe presentar un error de estado estacionario cero para un cambio de entrada escalón de lazo cerrado $\mathrm{y}_{\text {ref }}$ (alrededor del punto de operación) con una dinámica que se estabilice en un tiempo de 15 sg. con un sobreimpulso menor al $16.30 \%$.

\section{E. Diseño del controlador mediante el método del lugar geométrico de las raíces}

Considerando que para el controlador de procesos a utilizar (Hanyoung NX9), el tiempo integral TI que se puede configurar debe ser mayor a 1 sg. y no puede tener parte fraccionaria, iniciamos el diseño considerando un tiempo integral $\mathrm{Tl}=2$ sg. Así, utilizando la ecuación (3), se observa que se cuenta de inicio con un cero en el control de valor:

$a=\frac{1}{(\mathrm{TS} / \mathrm{TI}+1)}=0.8889$

El método para completar el diseño del controlador está basado en el lugar geométrico de las raíces el cual nos permitirá calcular la ganancia $\mathrm{K}$ del controlador de la ecuación (7). Con (8) en
(7) y el modelo discreto de la planta con $\mathrm{ZOH}$ dado por (6) obtenemos la siguiente función lugar geométrico de las raíces discreta:

$\mathrm{G}_{\mathrm{LGR}}(\mathrm{z})=\frac{0.061147(\mathrm{z}+2.414)(\mathrm{z}-0.8889)(\mathrm{z}+0.06473)}{\mathrm{z}(\mathrm{z}-1)(\mathrm{z}-0.5172)(\mathrm{z}-0.3672)}$

El lugar geométrico de las raíces discreta para esa función se muestra en la figura 14.

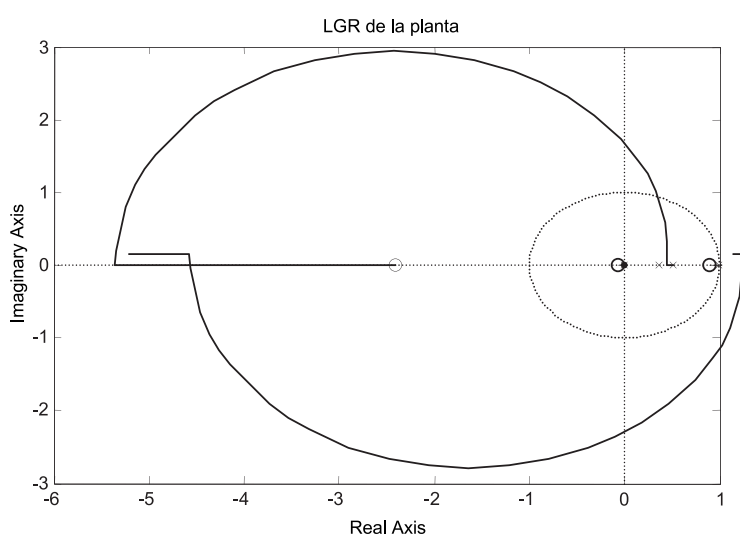

Fuente : Elaboración propia.

Figura 14. Lugar geométrico de las raíces discreta del lazo para $\mathrm{K}$ variable.

Debido a que la región cercana al círculo unitario será la dominante y corresponde a una respuesta sin sobreimpulso, se escoge el valor de ganancia tomando básicamente la especificación de tiempo de establecimiento. Esto corresponde a un valor de $\mathrm{K}=2.25$. Por tanto, el controlador discreto diseñado está representado por:

$G_{\mathrm{PI}}(\mathrm{z})=\mathrm{K} \frac{(\mathrm{z}-\mathrm{a})}{(\mathrm{z}-1)}=2.225 \frac{(\mathrm{z}-0.8889)}{(\mathrm{z}-1)}$

La respuesta esperada del sistema de lazo cerrado se muestra en la figura 15 . Se observa un leve sobreimpulso, un tiempo de establecimiento muy próximo al pedido y error cero a la entrada escalón de lazo cerrado; estas características de respuesta coinciden con las deseadas en las especificaciones de diseño.

\section{IMPLEMENTACIÓN DEL SISTEMA DE LAZO CE- RRADO}

De acuerdo a la ecuación (3), conocido el valor de la ganancia de control, la banda proporcional a con- 
figurar en el instrumento controlador de procesos Hanyoung NX9, se encuentra utilizando:

$P B=\frac{25 \times 16 \times 10^{-3}}{K} R(T S / T I+1)$

Con lo cual se obtiene un valor de $\mathrm{PB}=50$, el cual está en el rango configurable para este controlador industrial.

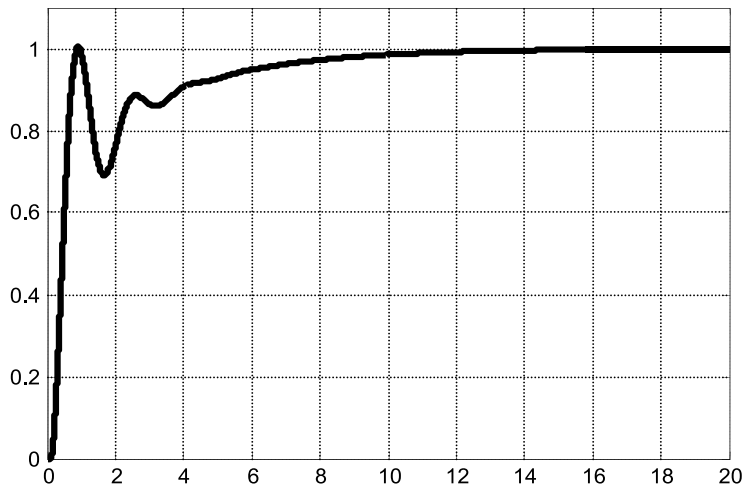

Fuente: Elaboración propia

Figura 15. Respuesta del sistema de lazo cerrado para el controlador diseñado, según diseño realizado.

La implementación de lazo cerrado se realiza de acuerdo al diagrama de bloques de la Figura 11, allí se ve la relación entre los parámetros configurables y la señal de referencia $y_{\text {ref }}$. Al inicio se configura el valor de MR para que permita a la planta iniciar con la salida en el valor del punto de operación. El valor ajustado es a MR $=75$ (equivale al $75 \%$ de la señal del control que entrega el controlador) (el algoritmo de control en base al error calcula la componente adicional de señal de control que se agrega a MR). Además, se configura el rango de entrada para recibir señales del proceso de 0 a 100 , esto se realiza configurando SLL a 0 y SLH a 100 . El valor inicial de SV se configura a 50 (esto significa que la referencia deseada en voltaje de salida es 3 voltios).

En la primera prueba realizada se produce un cambio en la referencia de lazo cerrado para pasar de 3 voltios a 2 voltios. Esto significa que luego de establecerse el punto de operación cambiamos el setpoint del instrumento a $\mathrm{SV}=25$ (equivale a desear que la salida llegue a 2 voltios). La respuesta debido al cambio escalón de referencia se muestra en la Figura 16. Además, se muestra el registro de la señal de control para esa prueba.
La siguiente prueba realizada consiste en cambiar el setpoint de entrada del último valor $S V=25$ a $\mathrm{SV}=50$ (lo que representa un cambio deseado de salida de 2 voltios a 3 voltios), el resultado de esa prueba se muestra en la figura 17. Se observa allí también la señal de control registrada en esa prueba.

Se prueba a continuación la compensación a perturbaciones del sistema de lazo cerrado. Manteniéndose la salida en SV = 50 (equivale a 3 voltios), se produce una perturbación que transitoriamente trata de disminuir la salida. En la Figura 18, se observa el cambio de la señal de control para compensar el efecto de la perturbación para luego de un transitorio lleve la salida nuevamente al valor deseado.

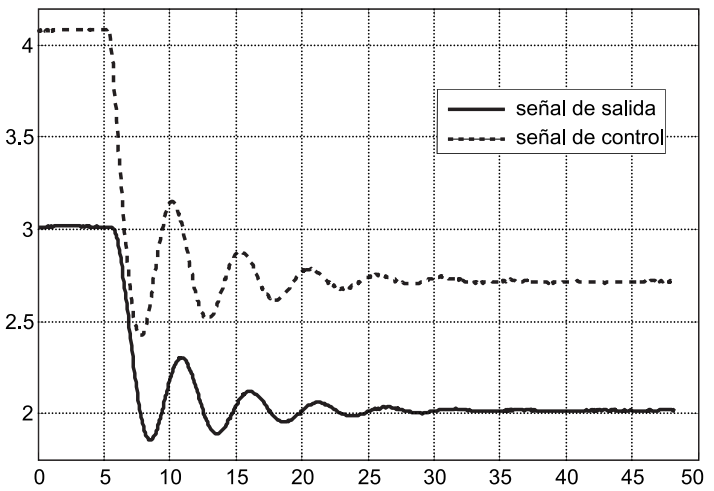

Fuente: Elaboración propia

Figura 16. Respuesta del sistema de lazo cerrado para un cambio de la referencia del tipo escalón de 3 voltios a 2 voltios.

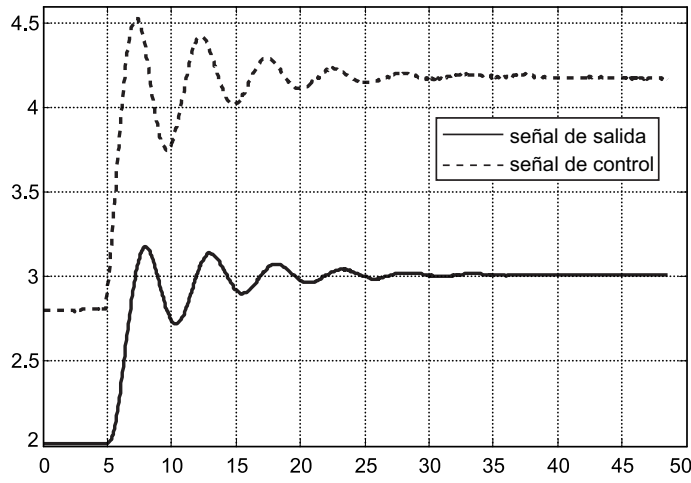

Fuente: Elaboración propia.

Figura 17. Respuesta del sistema de lazo cerrado para un cambio de la referencia del tipo escalón de 2 voltios a 3 voltios. 
Luego que la perturbación fue compensada y la salida regresa a su valor deseado, se produce otra señal de perturbación que en este caso trata de aumentar la salida. En la Figura 19, se observa el cambio de la señal de control para recuperar la salida al valor deseado.

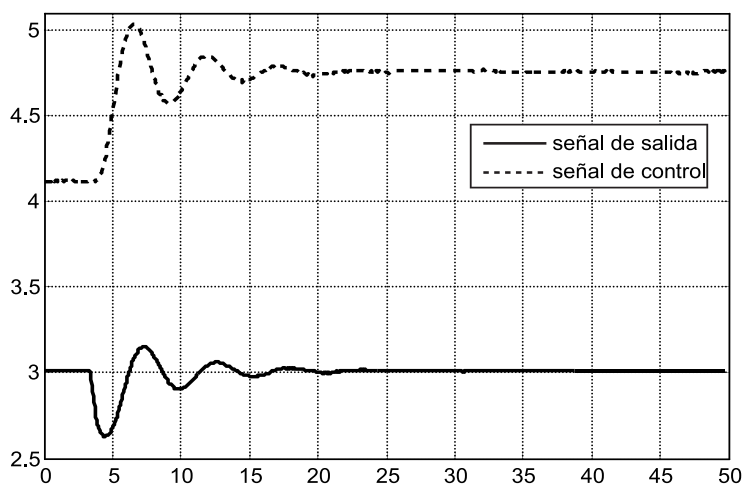

Fuente: Elaboración propia.

Figura 18. Cambio de la señal de control cuando se presenta una perturbación que intenta disminuir la salida.

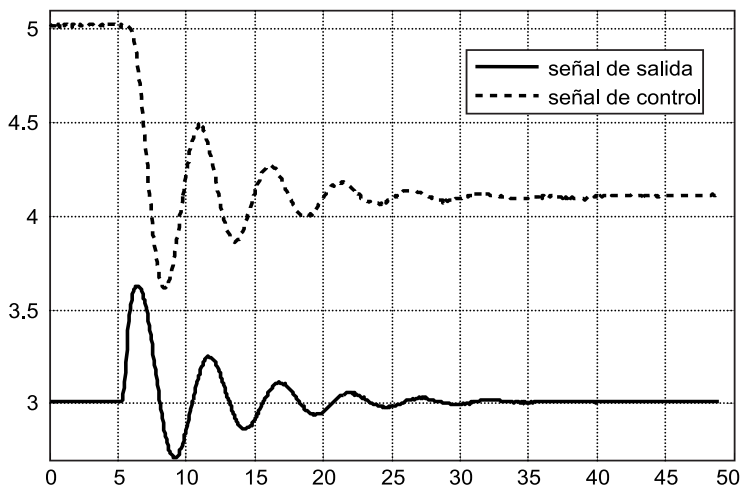

Fuente : Elaboración propia.

Figura 19. Cambio de la señal de control cuando se presenta una perturbación que intenta aumentar la salida.

\section{ANÁLISIS DE RESULTADOS}

En la Tabla I, se muestran los resultados en cuanto a los parámetros medidos de las respuestas a cambios escalón de la entrada de referencia de lazo cerrado de los diferentes controladores discretos diseñados e implementados en el controlador industrial Hanyoung NX9. El valor de entrada de referencia de lazo cerrado es de 3 voltios y es el mismo utilizado como punto de operación para la identificación de la planta (III.A.). Por ejemplo en la primera fila se muestran los valores medidos de las respuestas mostradas en las Figuras 16 y 17.

Todos los controladores sintonizados permiten obtener error de estado estacionario nulo para una entrada de lazo cerrado tipo escalón. Los tiempos de establecimiento obtenidos con los controladores diseñados son bastante cercanos a los especificados en el diseño, aunque los sobreimpulsos son levemente mayores.

Tabla I. Parámetros de la respuesta al escalón de la salida controlada por cada uno de los controladores diseñados.

\begin{tabular}{|c|c|c|c|c|}
\hline $\begin{array}{c}\text { Banda } \\
\text { Proporcional } \\
\text { (PB) }\end{array}$ & $\begin{array}{c}\text { Tiempo } \\
\text { Integral } \\
\text { (TI (sg.)) }\end{array}$ & $\begin{array}{c}\text { Error } \\
\text { en estado } \\
\text { estacionario }\end{array}$ & $\begin{array}{c}\text { Tiempo de } \\
\text { establecimiento } \\
\text { (sg.) }\end{array}$ & $\begin{array}{c}\text { Sobreimpulso } \\
\text { (Mp\%) }\end{array}$ \\
\hline 50 & 2 & 0 & 17 & 15 \\
\hline 50 & 3 & 0 & 20 & 0 \\
\hline 50 & 4 & 0 & 25 & 0 \\
\hline 50 & 5 & 0 & 35 & 0 \\
\hline
\end{tabular}

Fuente: Elaboración propia.

\section{CONCLUSIONES}

La metodología empleada permite diseñar controladores que se pueden implementar en un controlador industrial de procesos para obtener respuestas especificadas por los diseñadores, obteniéndose una buena aproximación de los resultados experimentales con los de simulación.

Resulta clave en esta metodología el proceso de identificación del modelo de la planta a ser controlada. Es muy importante validar el modelo con varias señales de prueba adicionales a las utilizadas en la identificación.

En razón que los parámetros de banda proporcional PB y tiempo de integración TI se deben configurar sólo en el rango permitido por el instrumento, es necesario iniciar el diseño sobretodo considerando un tiempo integral pequeño dentro del rango posible para evitar tiempos muy lentos de respuesta en lazo cerrado. Sin embargo, debe asegurarse en el diseño que las ramas del lugar geométrico adicionales a las dominantes no tengan una rápida salida del círculo unitario, pues el diseño resultará en sistemas inestables. De ser así, debemos aumentar el valor del TI inicial.

Es también muy importante verificar experimentalmente el cumplimiento de las relaciones mostradas en las Figuras 8, 9, 10 y 11; debido a que de no ser 
así, los resultados del diseño no se reflejarán en la implementación del sistema de lazo cerrado.

Por el carácter digital del instrumento, la metodología depende mucho del período de muestreo que utilice este controlador industrial a usar. En algunos casos ese dato no lo muestran los manuales, pero debe tratar de conseguir esa información para implementar esta propuesta.

\section{REFERENCIAS BIBLIOGRÁFICAS}

[1] Gene Franklin, David Powell, Abbas EmamiNaeini. Control de Sistemas Dinámicos con Retroalimentación. Addison-Wesley Iberoaméricana 1991.
[2] Katsuhiko Ogata. Ingeniería de Control Moderna. Prentice Hall Hispanoamericana S.A. 1993.

[3] Hanyoung Electronic Co. Ltd. Process Temperature Controller NX Series Instruction Manual, 2009.

[4] Karl Astrom, Bjorn Wittenmark. Computer Controlled Systems Theory and Design. Prentice Hall, Inc. 1997.

[5] Katsuhiko Ogata. Sistemas de Control en Tiempo Discreto. Prentice Hall Hispanoamericana S.A. 1996.

[6] http://www2.eie.ucr.ac.cr/ valfaro/docs/Vma. ucr.identificacion.metodo_123c.pdf 